\title{
1,8-Bis(diphenylamino)- and 1,8-Bis(methylphenylamino)naphthalene: Molecular Structure and Dynamic Behavior
}

\author{
Gösta Rimmler, Claus Krieger, and Franz A. Neugebauer* \\ Max-Planck-Institut für medizinische Forschung, Abteilung Organische Chemie, \\ Jahnstraße 29, W-6900 Heidelberg, F.R.G.
}

Received October 1, 1991

Key Words: 1,8-Diaminonaphthalenes, X-ray structure analysis of, conformational barriers of, dynamic behavior of

The title compounds 2 and 3 have been synthesized from 1,8diaminonaphthalene. The molecular structure of 2 has been determined by X-ray structure analysis and is discussed with regard to the arrangement of the peri-diphenylamino substit- uents in the crystalline state and the steric strain in the molecule. NMR studies of $\mathbf{3}$ reveal two conformational processes. Their nature is discussed and barriers are reported.
In diamines with the basic centers in close proximity the spatial interaction of the nitrogen lone pairs can lead to unusual molecular properties. The exceptionally high basicity of the "proton sponge" 1,8-bis(dimethylamino)naphthalene (1) results mainly from the destabilizing effect of the overlap of the nitrogen lone pairs and the steric strain in this diamine ${ }^{[1-3]}$. Radical cations of diamines can also be significantly stabilized when the close proximity of the nitrogen centers facilitates intramolecular 3-electron $\sigma$ bonding ${ }^{[4,5]}$. In this context we were interested in the properties of 1,8-bis(diphenylamino)naphthalene (2), particularly in the mutual influence of the two diphenylamino groups fixed at an $\mathrm{N} \cdots \mathrm{N}$ distance of about $280 \mathrm{pm}$. In this paper we report on the syntheses and properties of 1,8-bis(diphenylamino)- (2) and 1,8-bis(methylphenylamino)naphthalene (3). From 3 we expected to gain further insight into the conformational processes occurring in peri-substituted naphthalenes ${ }^{[6-9]}$.

A direct synthetic approach to $\mathbf{2}$ is the $\mathrm{N}$-phenylation of 1,8-diaminonaphthalene with iodobenzene in the presence of copper and a base. Preliminary experiments, however, showed that under these conditions only the phenyl-substituted $4(50 \%)$ and the two diphenyl derivatives $5(12 \%)$ and $6(1 \%)$ were formed. We therefore introduced the phenyl substituents in a stepwise procedure by starting from $1 \mathrm{H}$ perimidin-2(3H)-one (8). Thus, $N$-phenylation of 8 afforded the 1,3-diphenyl derivative 9 . Reductive ring cleavage of the latter with methyllithium provided 1,8-bis(phenylamino)naphthalene (6) which underwent reaction with dehydrobenzene to give 1-(diphenylamino)-8-(phenylamino)naphthalene (7). This compound proved to be rather unstable and decomposed readily under the required enforced reaction conditions. Therefore, $\mathrm{N}$-phenylation of 7 afforded the target 1,8-bis(diphenylamino)naphthalene (2) only in very low yield (ca. 1\%). A side-product isolated in $4 \%$ yield, $7 \mathrm{H}$ benz[kl]acridine (10), most likely arises from an intramolecular displacement reaction. 1,8-Bis(methylphenylamino)naphthalene (3) was obtained by methylation of 6 . The structures of these compounds were readily confirmed by NMR and mass spectra. An X-ray structure analysis of 2 revealed the arrangement of the diphenylamino groups in the crystalline state. Attempts, however, to obtain suitable crystals of $\mathbf{3}$ for an X-ray structure analysis were unsuccessful.<smiles>[R7]N([R7])c1cccc2cccc(N([R7])[Z7])c12</smiles>

\begin{tabular}{l|llll} 
& $\mathrm{R}^{1}$ & $\mathbf{R}^{2}$ & $\mathbf{R}^{3}$ & $\mathbf{R}^{4}$ \\
\hline $\mathbf{1}$ & $\mathrm{Me}$ & $\mathrm{Me}$ & $\mathrm{Me}$ & $\mathrm{Me}$ \\
$\mathbf{2}$ & $\mathrm{Ph}$ & $\mathrm{Ph}$ & $\mathrm{Ph}$ & $\mathrm{Ph}$ \\
$\mathbf{3}$ & $\mathrm{Me}$ & $\mathrm{Ph}$ & $\mathrm{Me}$ & $\mathrm{Ph}$ \\
$\mathbf{4}$ & $\mathrm{Ph}$ & $\mathrm{H}$ & $\mathrm{H}$ & $\mathrm{H}$ \\
$\mathbf{5}$ & $\mathrm{Ph}$ & $\mathrm{Ph}$ & $\mathrm{H}$ & $\mathrm{H}$ \\
$\mathbf{6}$ & $\mathrm{Ph}$ & $\mathrm{H}$ & $\mathrm{Ph}$ & $\mathrm{H}$ \\
7 & $\mathrm{Ph}$ & $\mathrm{Ph}$ & $\mathrm{Ph}$ & $\mathrm{H}$
\end{tabular}<smiles>[R]N1C(=O)N(F)c2cccc3cccc1c23</smiles><smiles>O=CC=CC=c1c2cccc3cccc(c32)n1-c1ccccc1</smiles>

10

Figure 1 shows the molecular crystal structure of $\mathbf{2}$ from a top view onto the naphthalene plane and from a side view along the $\mathrm{C}(8 \mathrm{a})-\mathrm{C}(4 \mathrm{a})$ bond which represents the axis of the approximate $C_{2}$ symmetry of the molecule. As in the bis(dimethylamino) analog $1^{[10]}$ the central $C(4 a)-C(8 a)$ bond is twisted [torsion angle $\mathrm{C}(1)-\mathrm{C}(8 \mathrm{a})-\mathrm{C}(4 \mathrm{a})-\mathrm{C}(4)$ : $\left.4.4^{\circ}, C(5)-C(4 a)-C(8 a)-C(8): 4.7^{\circ}\right]$. This torsion, however, is less pronounced than in $1\left(8.9^{\circ}\right)$. Accordingly, the distance of the nitrogen atoms from the least-squares plane through the naphthalene skeleton (mean value $25 \mathrm{pm}$ ) as well as the interplanar angles $\left[3.1^{\circ}\right.$, angle between $\mathrm{C}(2)-\mathrm{C}(3)-$ $C(4 a)-C(8 a)$ and $C(6)-C(7)-C(8 a)-C(4 a) ; 10.2^{\circ}, C(1)-$ $C(2)-C(8 a)$ and $C(7)-C(8)-C(8 a)]$ are significantly smaller than in 1 (mean value $40 \mathrm{pm}, 8.9^{\circ}$, and $20.1^{\circ}$ ). Surprisingly, 
the steric strain of the diphenylamino groups in peri-position leads only to a relatively small deviation of the naphthalene skeleton from planarity (Figure 1). On the other hand, the $\mathrm{N}\left(1^{\prime}\right)-\mathrm{C}(1)-\mathrm{C}(8 \mathrm{a})$ and $\mathrm{N}\left(8^{\prime}\right)-\mathrm{C}(8)-\mathrm{C}(8 \mathrm{a})$ angles of 2 (mean value $121.5^{\circ}$ ) are slightly larger than in 1 (mean value $120.5^{\circ}$ ) leading to an increase of the $\mathrm{N} \cdots \mathrm{N}$ distance in 2 $(285.8 \mathrm{pm})$ as compared to $1(279.2 \mathrm{pm})$. Additionally, the $\mathrm{N}-\mathrm{C}_{\mathrm{Naph}}$ distance is found to be a little larger (2: 142.9, 1 : $139.8 \mathrm{pm}$ ). As shown by the $\mathrm{C}-\mathrm{N}-\mathrm{C}$ angles (mean value, 2: $117.6,1: 115.7^{\circ}$ ) the nitrogen atoms in 2 are less pyramidal than in 1 , but still display a far from planar arrangement of the substituents. The nitrogen atoms in 2 lie about $23 \mathrm{pm}$ above the $\mathrm{C}_{\mathrm{Naph}}-\mathrm{C}_{\mathrm{Ph} 1}-\mathrm{C}_{\mathrm{Ph} 2}$ plane (1: $\left.30 \mathrm{pm}\right)$. Similar
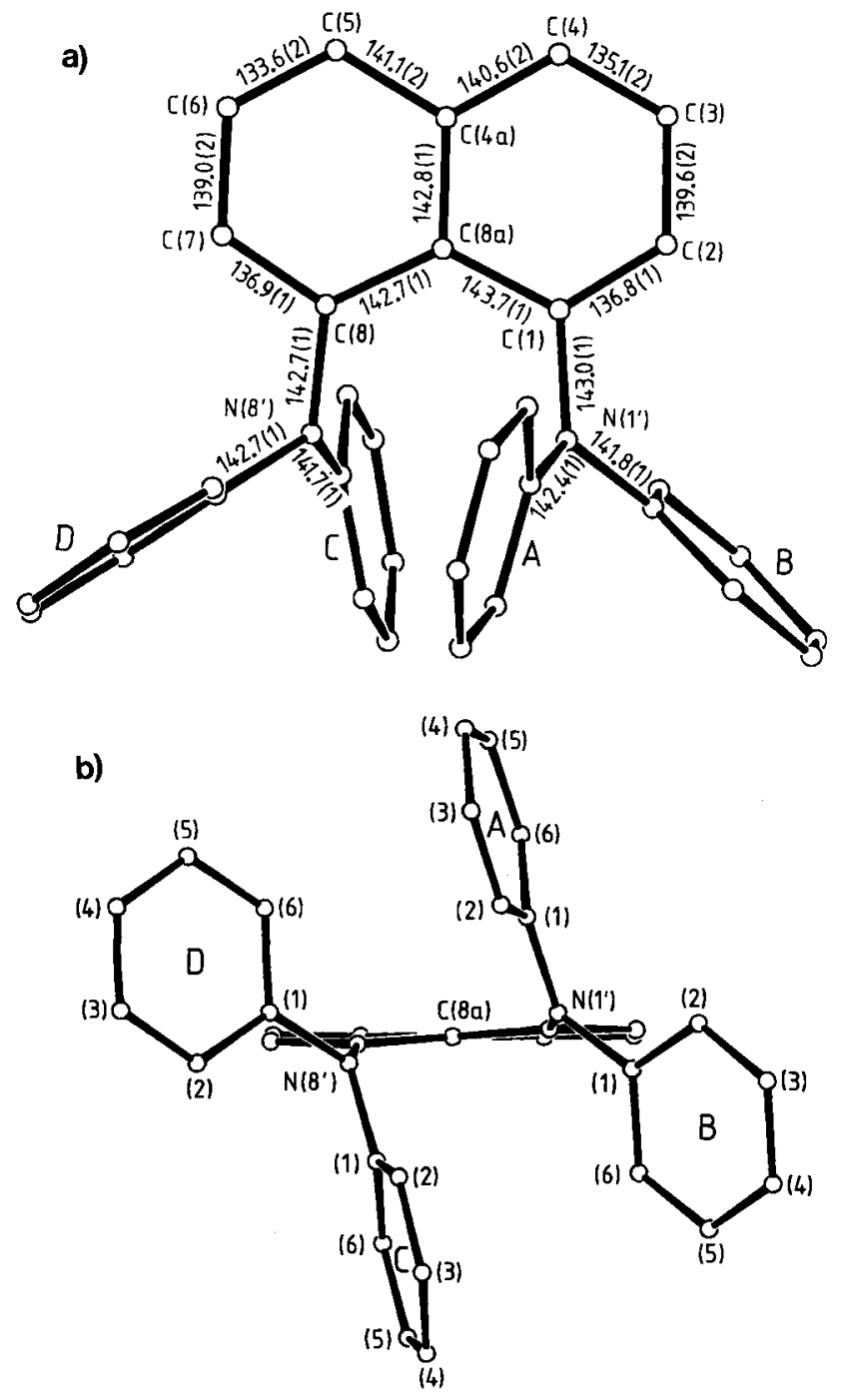

Figure 1. Molecular structure of 2: a) Top view showing bond distances [pm] of the 1,8-diaminonaphthalene part, b) side view across the naphthalene skeleton in the $\mathrm{C}(8 \mathrm{a})-\mathrm{C}(4 \mathrm{a})$ direction. Selected bond and torsional angles $\left[{ }^{\circ}\right]: \mathrm{C}(2)-\mathrm{C}(1)-\mathrm{N}\left(1^{\prime}\right)$ 118.0(1), $\mathrm{C}(8 \mathrm{a})-\mathrm{C}(1)-\mathrm{N}\left(1^{\prime}\right)$ 121.4(1), $\mathrm{C}(2)-\mathrm{C}(1)-\mathrm{C}(8 \mathrm{a})$ 120.6(1), $\mathrm{C}(7)-$ $\mathrm{C}(8)-\mathrm{N}\left(8^{\prime}\right) 118.1(1), \quad \mathrm{C}(8 \mathrm{a})-\mathrm{C}(8)-\mathrm{N}\left(8^{\prime}\right) 121.6(1), \mathrm{C}(7)-\mathrm{C}(8)-$ C(8a) 120.1(1), $\mathrm{C}(1)-\mathrm{N}\left(1^{\prime}\right)-\mathrm{C}(1 \mathrm{~A})$ 116.4(1), $\mathrm{C}(1)-\mathrm{N}\left(1^{\prime}\right)-\mathrm{C}(1 \mathrm{~B})$ $118.0(1), \quad \mathrm{C}(1 \mathrm{~A})-\mathrm{N}\left(1^{\prime}\right)-\mathrm{C}(1 \mathrm{~B}) \quad 120.0(1), \quad \mathrm{C}(8)-\mathrm{N}\left(8^{\prime}\right)-\mathrm{C}(1 \mathrm{C})$ $116.5(1), \quad C(8)-N\left(8^{\prime}\right)-C(1 D) \quad 116.4(1), \quad C(1 C)-N\left(8^{\prime}\right)-C(1 D)$ 118.2(1); $\mathrm{C}(2)-\mathrm{C}(1)-\mathrm{N}\left(1^{\prime}\right)-\mathrm{C}(1 \mathrm{~A}) \quad 106.9(2), \mathrm{C}(2)-\mathrm{C}(1)-\mathrm{N}\left(1^{\prime}\right)-$ $\mathrm{C}(1 \mathrm{~B})-46.7(2), \mathrm{C}(7)-\mathrm{C}(8)-\mathrm{N}\left(8^{\prime}\right)-\mathrm{C}(1 \mathrm{C}) 104.7(2), \mathrm{C}(7)-\mathrm{C}(8)-$ $N\left(8^{\prime}\right)-C(1 D)-42.2(2)$
$\mathrm{N}-\mathrm{C}_{\mathrm{Naph}}$ and $\mathrm{N}-\mathrm{C}_{\mathrm{Ph}}$ bonds $(142.1-142.8 \mathrm{pm})$ indicate an equal interaction of the nitrogen lone pairs with the three aryl substituents giving all $\mathrm{N}-\mathrm{C}$ bonds some $\pi$ character. In 1 the $\mathrm{N}-\mathrm{C}_{\mathrm{Naph}}$ distance is shortened $(139.8 \mathrm{pm}$, corresponding to about $20 \%$ double bond character $\left.{ }^{[10]}\right)$ which seems to contribute to the steric strain in the "proton sponge" 1 . Another contribution to the steric strain in 1 arises from the destabilizing overlap of the nitrogen lone pairs. To minimize this interaction 1 adopts a conformation such that one methyl group of each amino substituent lies almost in the naphthalene plane (mean torsional angle $162^{\circ}$ ). In 2 this interaction is apparently less effective due to the enhanced delocalization of the lone pairs into the aryl substituents. The corresponding torsional angle [mean of $\mathrm{C}(8 \mathrm{a})-\mathrm{C}(8)-\mathrm{N}\left(8^{\prime}\right)-\mathrm{C}(1 \mathrm{D})$ and $\mathrm{C}(8 \mathrm{a})-\mathrm{C}(1)-\mathrm{N}\left(1^{\prime}\right)-$ $\mathrm{C}(1 \mathrm{~B})]$ is considerably smaller (mean value $140^{\circ}$ ). 2 appears to accomodate the bulky peri-diphenylamino groups with surprisingly little strain.

These results on $\mathbf{2}$ in its solid state correlate well with its dynamic behavior in solution. In the ${ }^{1} \mathrm{H}-\mathrm{NMR}$ spectra of 2 at ambient temperature all corresponding phenyl protons (ortho, meta, para) appear to be equivalent indicating conformational equilibria, i.e. a fast rotation about the $\mathrm{N}-\mathrm{C}_{\mathrm{Ph}}$ bonds and a fast ring flipping of the naphthalene skeleton. A third possibility, a rotation about the $\mathrm{N}-\mathrm{C}_{\mathrm{Naph}}$ bonds, is unlikely for steric reasons. Below $263 \mathrm{~K}$ the doublet of the ortho-phenyl protons broadens, disappearing at $213 \mathrm{~K}$. Line broadening of the other proton signals sets in below $200 \mathrm{~K}$. NMR studies at temperatures lower than $200 \mathrm{~K}$, however, were hampered by the low solubility of 2 . The observed line broadening of 2 indicates that the barriers of the involved
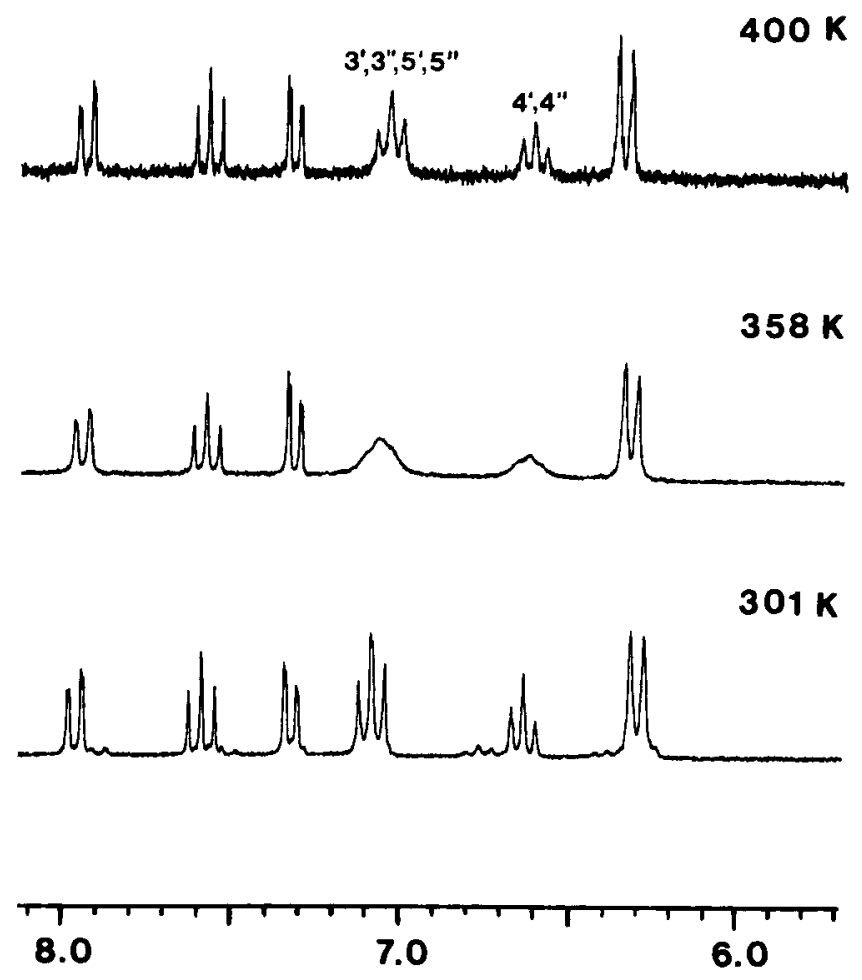

Figure 2. ${ }^{1} \mathrm{H}-\mathrm{NMR}$ spectra of 3 in $\left[\mathrm{D}_{6}\right] \mathrm{DMSO}$ at 400,358 , and $301 \mathrm{~K}(200 \mathrm{MHz}) ; \delta$ scale 

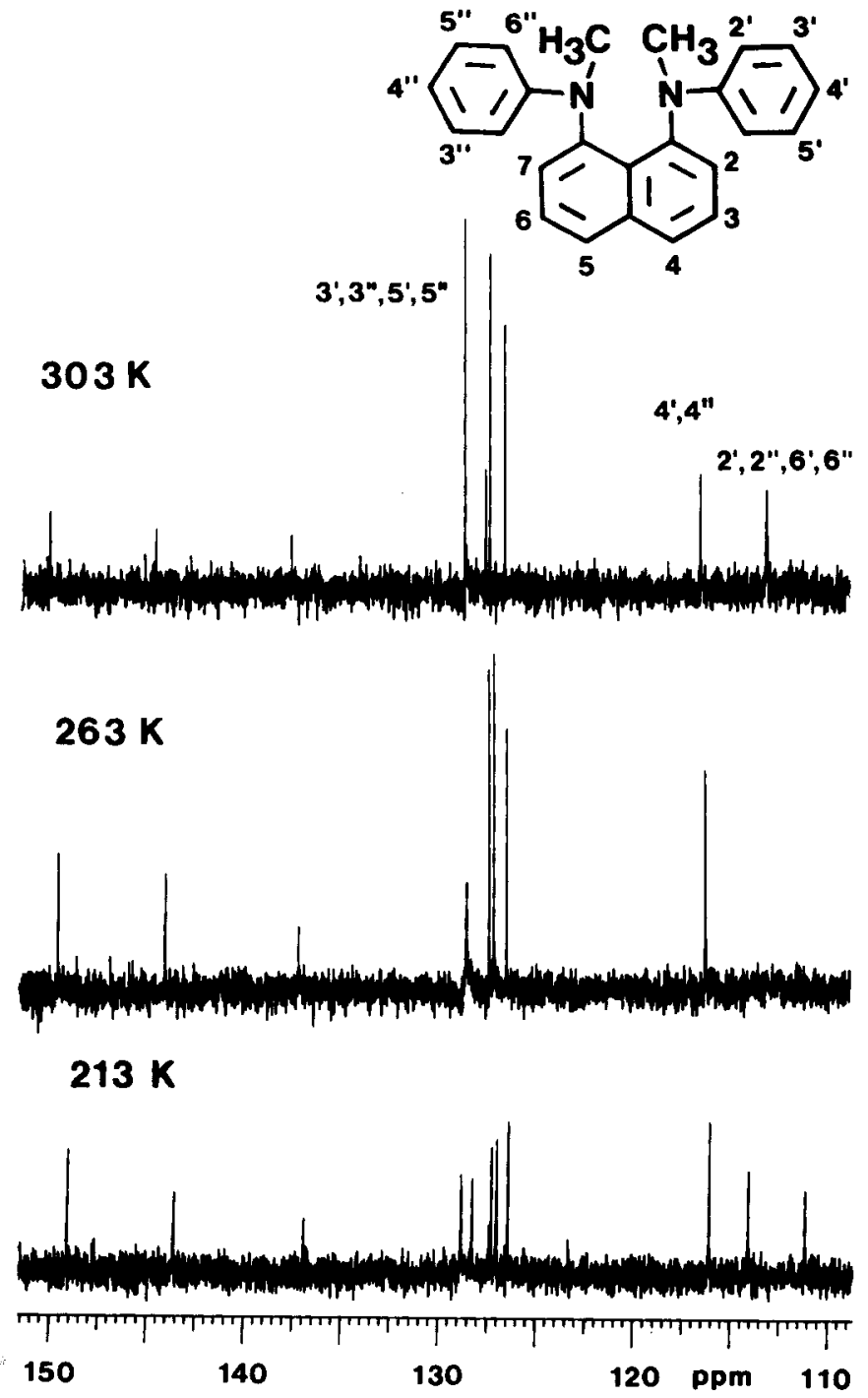

Figure $3 .{ }^{13} \mathrm{C}-\mathrm{NMR}$ spectra of 3 in $\mathrm{CDCl}_{3}$ at 303,263 , and $213 \mathrm{~K}$ $(125.8 \mathrm{MHz}) ; \delta$ scale

conformational processes, ring flipping and rotation about the $\mathrm{N}-\mathrm{C}_{\mathrm{Ph}}$ bonds, can be considered to be $<10 \mathrm{kcal} \mathrm{mol}^{-1}$.

A significantly different behavior is observed for the related 1,8-bis(methylphenylamino)naphthalene (3). At ambient temperature the ${ }^{1} \mathrm{H}$-NMR spectrum of 3 shows the presence of two conformers, $3 \mathbf{a}$ and $\mathbf{3 b}$, in a ratio of about $91: 9$ which interconvert at higher temperatures (Figure 2). In addition, as the temperature is lowered, specific ${ }^{1} \mathrm{H}$ and ${ }^{13} \mathrm{C} \mathrm{N}$ phenyl signals of the major conformer broaden and clearly split below $240 \mathrm{~K}$ (Figure 3). These spectral changes of 3 suggest that two conformational processes are taking place. The ${ }^{1} \mathrm{H}-\mathrm{NMR}$ data of both conformers were determined and assigned on the basis of 2D-(H,H)-COSY-90 and NOE experiments and are collected in Table 1. In the ${ }^{13} \mathrm{C}$-NMR spectra only signals of the major conformer $\mathbf{3 a}$ are clearly detected (Figure 3). All NMR results of both conformers point to molecular $C_{2}$ symmetry about $\mathrm{C}(4 \mathrm{a})-\mathrm{C}(8 \mathrm{a})$.

One can reasonably assume that the barrier to ring flipping of the naphthalene skeleton (compare Figure 1b) will be close to that of $1(7.5 \mathrm{kcal} / \mathrm{mol})$ and $2(<10 \mathrm{kcal} / \mathrm{mol})$. Hence the observed high-temperature interconversion of 3a and $\mathbf{3 b}$ is likely to correspond to a rotation about the $\mathrm{N}-\mathrm{C}_{\mathrm{Naph}}$ bond via a transition state where the dynamic plane of the $\mathrm{N}$ atom is coplanar to that of the naphthalene skeleton.

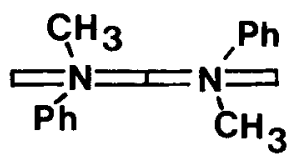

$3 \mathbf{a}$

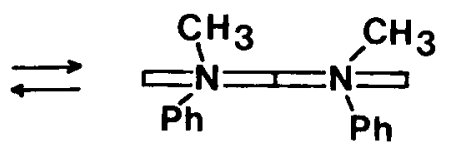

$3 \mathbf{b}$
A comparison of the ${ }^{1} \mathrm{H}-\mathrm{NMR}$ data in Table 1 shows that the corresponding naphthalene protons of $\mathbf{3 a}$ and $\mathbf{3 b}$ exhibit similar chemical shifts. Distinct differences, however, were found for the two sets of methyl and phenyl protons which helped in the assignment of the conformers. The methyl groups of 3a, which are close to the adjacent phenyl substituents, are expected to show up at higher field $(\delta \approx 2.7)$ than those of $\mathbf{3 b}(\delta \approx 3.3)$. Similarly, due to the mutual shielding of the syn-arranged phenyl groups, the signals of the phenyl protons in $\mathbf{3 b}$ should appear at somewhat higher field than those of $\mathbf{3 a}$.

Table 1. ${ }^{1} \mathrm{H}$-chemical shifts $(\delta)$ of $\mathbf{3 a}$ and $\mathbf{3 b}$ at $303 \mathrm{~K}(500 \mathrm{MHz})$

\begin{tabular}{lllll}
\hline & \multicolumn{2}{c}{$\mathrm{CDCl}_{3}$} & \multicolumn{2}{c}{$\left[\mathrm{D}_{6}\right] \mathrm{DMSO}$} \\
Protons & 3a & 3b & 3a & 3b \\
\hline $\mathrm{CH}_{3}$ & 2.71 & 3.30 & 2.61 & 3.23 \\
$2^{\prime}, 2^{\prime \prime}, 6^{\prime}, 6^{\prime \prime}$ & 6.40 & 6.28 & 6.28 & 6.25 \\
$4^{\prime}, 4^{\prime \prime}$ & 6.66 & 6.4 & 6.62 & 6.37 \\
$3^{\prime}, 3^{\prime \prime}, 5^{\prime}, 5^{\prime \prime}$ & 7.10 & 6.79 & 7.07 & 6.75 \\
2,7 & 7.29 & 7.3 & 7.30 & 7.29 \\
3,6 & 7.48 & 7.44 & 7.58 & 7.52 \\
4,5 & 7.82 & 7.78 & 7.95 & 7.88 \\
\hline
\end{tabular}

The separate signals of related protons of $\mathbf{3 a}$ and $\mathbf{3 b}$ at ambient temperature (Table 1, Figure 2) broaden on heating and coalesce at different temperatures, depending on the shift difference, thus confirming that the pairs of signals are due to conformers of a single compound. Particularly the $4^{\prime}, 4^{\prime \prime}$ and $3^{\prime}, 3^{\prime \prime}, 5^{\prime}, 5^{\prime \prime}$ protons exhibit these changes (Figure 2). The coalescence of the $\mathrm{CH}_{3}$ proton signals, however, cannot be clearly observed, since at higher temperatures the $\mathrm{H}_{2} \mathrm{O}$ resonance superimposes. We evaluated the barriers to the exchange process by two methods. The Shanan-Atidi and Bar-Eli approximation ${ }^{[1]]}$ yields $\Delta G^{*}=19.3 \mathrm{kcal} / \mathrm{mol}$ for the process $3 \mathbf{a} \rightarrow \mathbf{3 b}$ and $\Delta G^{*}=17.7 \mathrm{kcal} / \mathrm{mol}$ for $\mathbf{3 b} \rightarrow$ 3a (Table 2). Slightly different free energies of activation were obtained by the approximation of Anet and Basus ${ }^{[12]}$ for the exchange between two species of very unequal populations, $18.6 \mathrm{kcal} / \mathrm{mol}$ for $\mathbf{3 a} \rightarrow \mathbf{3 b}$ and $17.0 \mathrm{kcal} / \mathrm{mol}$ for $\mathbf{3 b} \rightarrow \mathbf{3 a}$. The free energy difference between $\mathbf{3 a}$ and $\mathbf{3 b}$ is $1.6 \mathrm{kcal} / \mathrm{mol}$. The higher energy of $\mathbf{3 b}$ is undoubtedly the 
result of steric strain due to the syn-arrangement of both phenyl substituents. Based on the obtained results the observed high-temperature process of 3 is interpreted to be the transformation $\mathbf{3 a} \rightleftharpoons \mathbf{3} \mathbf{b}$ caused by a rotation about the $\mathrm{C}-\mathrm{N}_{\mathrm{Naph}}$ bond. A similar barrier $\left(\Delta G^{+}=13.7 \mathrm{kcal} / \mathrm{mol}\right)$ was reported for 1-(benzylmethylamino)-8-(dimethylamino)naphthalene ${ }^{[6]}$.

Table 2. Free energies of activation $\Delta G^{\neq}$for the conformational processes in $\mathbf{3}$

\begin{tabular}{|c|c|c|c|c|}
\hline & Process & $\Delta \nu[\mathrm{Hz}]$ & $\mathrm{T}_{\mathrm{c}}[\mathrm{K}]$ & $\Delta \mathrm{G}^{*}\left[\mathrm{kcal} \cdot \mathrm{mol}^{-1}\right]$ \\
\hline $4^{\prime}, 4^{\prime \prime}-\mathrm{H}^{[\mathrm{a}]}$ & $\mathbf{3 a} \rightarrow \mathbf{3 b}$ & 65 & $358^{[c]}$ & $19.2^{[\mathrm{e}]}$ \\
\hline $3^{\prime}, 3^{\prime \prime}, 5^{\prime}, 5^{\prime \prime}-H^{[a]}$ & $\mathbf{3 a} \rightarrow \mathbf{3 b}$ & 50 & $358^{[c]}$ & $19.4^{[e]}$ \\
\hline $4^{\prime}, 4^{\prime \prime} \cdot \mathrm{H}^{[a]}$ & $3 a \rightarrow 3 b$ & 65 & $358^{[c]}$ & $18.5^{[\mathrm{f}]}$ \\
\hline $3^{\prime}, 3^{\prime \prime}, 5^{\prime}, 5^{\prime \prime}-\mathrm{H}^{[a]}$ & $3 \mathbf{a} \rightarrow \mathbf{3 b}$ & 50 & $358^{[c]}$ & $18.7^{[\mathrm{f}]}$ \\
\hline $4^{\prime}, 4^{\prime \prime}-\mathrm{H}^{[\mathrm{a}]}$ & $\mathbf{3 b} \rightarrow \mathbf{3 a}$ & 65 & $358^{[\mathrm{c}]}$ & $17.6^{[\mathrm{e}]}$ \\
\hline $3^{\prime}, 3^{\prime \prime}, 5^{\prime}, 5^{\prime \prime}-\mathrm{H}^{[\mathrm{a}]}$ & $3 \mathbf{b} \rightarrow 3 \mathbf{a}$ & 50 & $358^{[\mathrm{c}]}$ & $17.7^{[\mathrm{e}]}$ \\
\hline $4^{\prime}, 4^{\prime \prime}-\mathrm{H}^{[\mathrm{a}]}$ & $\mathbf{3 b} \rightarrow \mathbf{3 a}$ & 65 & $358^{[\mathrm{c}]}$ & $16.9^{[f]}$ \\
\hline $3,3^{\prime \prime}, 5^{\prime}, 5^{\prime \prime}-\mathrm{H}^{[a]}$ & $\mathbf{3 b} \rightarrow \mathbf{3 a}$ & 50 & $358^{[\mathrm{c}]}$ & $17.1^{[\mathrm{f}]}$ \\
\hline $2^{\prime}, 2^{\prime \prime}, 6^{\prime}, 6^{\prime \prime}-H^{[b]}$ & N-C Ph $_{\text {Rot. }}$. & 200 & $256^{[\mathrm{d}]}$ & $11.8^{[\mathrm{g}]}$ \\
\hline 3',3",5',5"--H[b] & N-C Ph $_{\mathbf{P h}}$ Rot. & 165 & $252^{[d]}$ & $11.7[8]$ \\
\hline $2^{\prime}, 2^{\prime \prime}, 6^{\prime}, 6^{\prime \prime}-13 \mathrm{C}^{\mathrm{b}]}$ & N-C $\mathrm{Ph}_{\mathrm{Ph}}$ Rot. & 370 & $265^{[\mathrm{d}]}$ & $11.9[\mathrm{~g}]$ \\
\hline
\end{tabular}

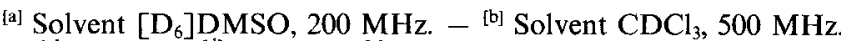
$-{ }^{[c]} \pm 5 K .{ }^{[d]} \pm 2 K$. - ${ }^{[\mathrm{e}]}$ Evaluated by means of the ShananAtidi and Bar-Eli approximation ${ }^{[11]}$ - ${ }^{[1]}$ Evaluated by means of the Anet and Basus approximation ${ }^{[12]}$ - ${ }^{[\mathrm{g}]}$ Evaluated by means of the Gutowsky and Holm approximation ${ }^{[13]}$.

In addition to the $\mathrm{C}-\mathrm{N}_{\text {Naph }}$ torsional process of $\mathbf{3}$ a second motion, corresponding to the rotation about the $\mathrm{N}-\mathrm{C}_{\mathrm{Ph}}$ bond, can be detected. At lower temperature the signals of the ortho $\left(2^{\prime}, 2^{\prime \prime}, 6^{\prime}, 6^{\prime \prime}\right)$ and meta $\left(3^{\prime}, 3^{\prime \prime}, 5^{\prime}, 5^{\prime \prime}\right)$ phenyl protons as well as those of the corresponding ring carbons of 3a (Figure 3) broaden and clearly split to pairs of signals indicating a "frozen" $\mathrm{N}-\mathrm{C}_{\mathrm{Ph}}$ rotation. The free energy of activation of the process was calculated by the Gutowsky and Holm approximation ${ }^{[13]}$ to be $11.7 \pm 0.2 \mathrm{kcal} / \mathrm{mol}$. This value is considerably larger than the $\mathrm{N}-\mathrm{C}_{\mathrm{Ph}}$ rotation barrier found for 1-(methylphenylamino)naphthalene $\left(\Delta G^{*}=\right.$ $\left.7.1 \mathrm{kcal} / \mathrm{mol}, T_{\mathrm{c}}=153 \mathrm{~K}\right)^{[9]}$. Thus, the steric strain of 3, introduced by the bulky substituents in the peri-positions, also raises the barrier to $\mathrm{N}-\mathrm{C}_{\mathrm{Ph}}$ rotation.

Attempts to characterize the radical cations of 2 and 3 by ESR under stationary conditions failed due to the short lifetime of these species. This is surprising, since diarylamine and triarylamine radical cations can be easily studied by $\operatorname{ESR}^{[14,15]}$. The short lifetime of $\mathbf{2}^{+\bullet}$ and $\mathbf{3}^{+\bullet}$ may be caused by the high chemical reactivity in the positions 4 and 5 . Radical cations of 1,8-diamino-substituted naphthalenes without substituents in the 4,5-positions have not yet been identified by ESR ${ }^{[16]} .2$ and 3 also show no unusual basic properties.
We thank the Deutsche Forschungsgemeinschaft for support of this work.

\section{Experimental}

Meiting points (uncorrected): Büchi apparatus. - UV: Cary 2300 spectrophotometer. $-{ }^{1} \mathrm{H}$ and ${ }^{13} \mathrm{C}$ NMR: Bruker AC-200, Bruker HX-360 or AM- 500 spectrometer at $303 \mathrm{~K}$, unless stated otherwise (with tetramethylsilane as internal standard). - MS: Dupont CEC 21-492 (70 eV) or Finnigan MAT $212(70 \mathrm{eV})$ spectrometer. - Analytical TLC plates: Macherey-Nagel Polygram SIL G/UV 254 - Preparative column chromatography: Woelm silica gel (0.063-0.200 mm). - MPLC column: Reversed phase HD-SIL18-30-60.

$X$-ray Crystal Structure Analysis of $2^{[17]}$ : Colorless crystals from hexane. Crystal data: $\mathrm{C}_{34} \mathrm{H}_{26} \mathrm{~N}_{2}$, molecular mass 462.21 , crystal size $0.25 \times 0.2 \times 0.1 \mathrm{~mm}$, triclinic, space group $P \overline{1}, a=1006.7(2), b=$ 1078.6(2), $c=1250.8(3) \mathrm{pm}, \alpha=68.86(2), \beta=78.47(2), \gamma=$ $82.61(2)^{\circ}, Z=2, d_{\text {calcd. }}=1.240 \mathrm{gcm}^{-3}$. Data collection: Enraf-Nonius CAD-4 four-circle diffractometer, graphite-monochromated Mo- $K_{\alpha}$ radiation ( $\lambda=71.069 \mathrm{pm}, \Theta / 2 \Theta$ scanning technique), 5961 independent reflections $\left(\max , \sin \Theta / \lambda=6.6 \mathrm{~nm}^{-1}\right)$; absorption correction was not necessary, $\mu\left(\mathrm{Mo}-K_{\alpha}\right)=0.671 \mathrm{~cm}^{-1}$. Structure solution and refinement: Structure solution by direct methods (MULTAN), refinement by full matrix technique of $F^{2}$ by using anisotropic temperature factors for non-hydrogen atoms and isotropic temperature factors for hydrogen ${ }^{[18]}$. Atomic scattering factors and anomalous dispersion corrections were taken from International Tables for X-Ray Crystallography ${ }^{[19]} \cdot R=0.040\left(R_{w}=0.039\right)$ for 3591 reflections $[I \geqslant 3 \sigma(I)]$; residual electron density $\Delta \mathrm{Q}=120 \mathrm{e}$ $\mathrm{nm}^{-3}$. Positional and thermal parameters of non-hydrogen atoms are given in Table 3.

Phenylation of 1,8-Diaminonaphthalene in Diphenyl Ether: A vig-

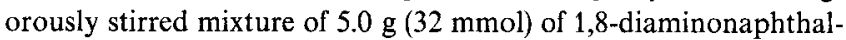

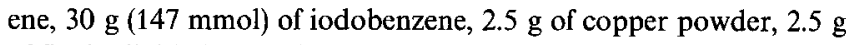
of finely divided potassium carbonate, and a trace of iodine in $30 \mathrm{~g}$ of diphenyl ether was heated at reflux for $2 \mathrm{~d}$. After the excess iodobenzene and solvent were removed under high vacuum, the remaining residue was extracted several times with dichloromethane. The combined filtered extracts were concentrated under reduced pressure and chromatographed [silica gel, cyclohexane/toluene $(3: 1)$ followed by dichloromethane] to yield three products:

1-Amino-8-(diphenylamino) naphthalene (5): $R_{\mathrm{f}}=0.44$ [cyclohexane/toluene (3:1)]; $1.20 \mathrm{~g}(12 \%)$ of reddish colored crystals from ethanol, m.p. $162^{\circ} \mathrm{C}$. - IR $\left(\mathrm{CHCl}_{3}\right): \tilde{v}=3495 \mathrm{~cm}^{-1}, 3395\left(\mathrm{NH}_{2}\right)$. - UV (dioxane): $\lambda_{\max }(\lg \varepsilon)=292 \mathrm{~nm}(4.16), 350$ (4.04). - ${ }^{\mathrm{t}} \mathrm{H}$ NMR $\left(\mathrm{CDCl}_{3}, 500 \mathrm{MHz}\right): \delta=5.20\left(\mathrm{~s}, 2 \mathrm{H}, \mathrm{NH}_{2}\right), 6.55\left(\mathrm{dd},{ }^{3} \mathrm{~J}=5.8,{ }^{4} \mathrm{~J}=\right.$ $2.9 \mathrm{~Hz}, 1 \mathrm{H}, 7-\mathrm{H}), 6.95\left(\mathrm{dd},{ }^{3} J \approx{ }^{3} J^{\prime} \approx 7.5 \mathrm{~Hz}, 2 \mathrm{H}, 4^{\prime}, 4^{\prime \prime}-\mathrm{H}\right), 7.03$ $\left(\mathrm{d},{ }^{3} J=8.0 \mathrm{~Hz}, 4 \mathrm{H}, 2^{\prime}, 2^{\prime \prime}, 6^{\prime}, 6^{\prime \prime}-\mathrm{H}\right), 7.07\left(\mathrm{~d},{ }^{3} J=7.9 \mathrm{~Hz}, 1 \mathrm{H}, 2-\mathrm{H}\right)$, $7.20\left(\mathrm{dd},{ }^{3} J \approx{ }^{3} J^{\prime} \approx 8.0 \mathrm{~Hz}, 4 \mathrm{H}, 3^{\prime}, 3^{\prime \prime}, 5^{\prime}, 5^{\prime \prime}-\mathrm{H}\right), 7.20-7.25(\mathrm{~m}, 2 \mathrm{H}$, $5,6-\mathrm{H}), 7.35\left(\mathrm{dd},{ }^{3} J \approx{ }^{3} J^{\prime} \approx 7.8 \mathrm{~Hz}, 1 \mathrm{H}, 3-\mathrm{H}\right), 7.69\left(\mathrm{~d},{ }^{3} J=8.1 \mathrm{~Hz}\right.$, $1 \mathrm{H}, 4-\mathrm{H}$ ). - MS: high resolution $\left[\mathrm{M}^{+}\right], m / z=310.1477$ (calcd. $310.1470) ; m / z(\%)=310(100)\left[\mathrm{M}^{+}\right], 217(30)$.

\section{$\mathrm{C}_{22} \mathrm{H}_{18} \mathrm{~N}_{2}$ (310.4) Calcd. C 85.13 H 5.85 N 9.03 Found C 84.96 H 5.94 N 9.03}

1,8-Bis (phenylamino) naphthalene (6): $R_{\mathrm{f}}=0.2$ [cyclohexane/toluene (3:1)]; $90 \mathrm{mg}(1 \%)$ of colorless needles from ethanol, m.p. $147^{\circ} \mathrm{C}$. IR $\left(\mathrm{CHCl}_{3}\right): \tilde{v}=3370 \mathrm{~cm}^{-1}, 3295(\mathrm{NH})$. - UV (dioxane): $\lambda_{\max }(\lg \varepsilon)=355 \mathrm{~nm}(4.10) .-{ }^{1} \mathrm{H} \mathrm{NMR}\left(\mathrm{CDCl}_{3}, 500 \mathrm{MHz}\right): \delta=$ $6.90\left(\mathrm{dd},{ }^{3} J \approx{ }^{3} J^{\prime} \approx 7.3 \mathrm{~Hz}, 2 \mathrm{H}, 4^{\prime}, 4^{\prime \prime}-\mathrm{H}\right), 6.91\left(\mathrm{~d},{ }^{3} J=7.0 \mathrm{~Hz}, 4 \mathrm{H}\right.$, $\left.2^{\prime}, 2^{\prime \prime}, 6^{\prime}, 6^{\prime \prime}-\mathrm{H}\right), 7.20\left(\mathrm{dd},{ }^{3} J \approx{ }^{3} J^{\prime} \approx 8.2 \mathrm{~Hz}, 4 \mathrm{H}, 3^{\prime}, 3^{\prime \prime}, 5^{\prime}, 5^{\prime \prime}-\mathrm{H}\right), 7.22$ $\left(\mathrm{dd},{ }^{3} J=6.7,{ }^{4} J=0.8 \mathrm{~Hz}, 2 \mathrm{H}, 2,7-\mathrm{H}\right), 7.33\left(\mathrm{dd},{ }^{3} J \approx{ }^{3} J^{\prime} \approx 7.8 \mathrm{~Hz}\right.$, 
Table 3. Fractional atomic coordinates and equivalent isotropic thermal parameters $\left[\mathrm{pm}^{2}\right]$ for non-hydrogen atoms of 2 with e.s.d.'s in the least significant figure in parentheses; $U_{\mathrm{eq}}=1 / 3 \sum_{i} \sum_{i} U_{i j} a_{i}^{*} a_{j}^{*} \mathbf{a}_{i} \cdot \mathbf{a}_{j}$

\begin{tabular}{|c|c|c|c|c|}
\hline & $\mathrm{x}$ & $\mathbf{y}$ & $\mathrm{z}$ & $\mathrm{U}_{\mathrm{eq}}$ \\
\hline$C(1)$ & $0.2121(1)$ & $0.1051(1)$ & $0.6625(1)$ & $419(5)$ \\
\hline$C(2)$ & $0.1626(2)$ & $0.0537(1)$ & $0.5941(1)$ & $517(6)$ \\
\hline$C(3)$ & $0.1008(2)$ & $-0.0675(1)$ & $0.6404(1)$ & $605(6)$ \\
\hline $\mathrm{C}(4)$ & $0.0849(2)$ & $-0.1337(1)$ & $0.7562(1)$ & $621(6)$ \\
\hline$C(4 a)$ & $0.1352(1)$ & $-0.0867(1)$ & $0.8307(1)$ & $503(6)$ \\
\hline$C(5)$ & $0.1150(2)$ & $-0.1578(1)$ & $0.9514(1)$ & $661(7)$ \\
\hline$C(6)$ & $0.1601(2)$ & $-0.1149(2)$ & $1.0246(1)$ & $706(7)$ \\
\hline$C(7)$ & $0.2356(2)$ & $-0.0028(1)$ & $0.9815(1)$ & $583(6)$ \\
\hline $\mathrm{C}(8)$ & $0.2615(1)$ & $0.0689(1)$ & $0.8651(1)$ & $439(5)$ \\
\hline$C(8 a)$ & $0.2055(1)$ & $0.0331(1)$ & $0.7849(1)$ & $412(5)$ \\
\hline$N\left(1^{\prime}\right)$ & $0.2616(1)$ & $0.2356(1)$ & $0.6102(1)$ & $415(4)$ \\
\hline $\mathbf{N}\left(8^{\prime}\right)$ & $0.3538(1)$ & $0.1722(1)$ & $0.8266(1)$ & $434(4)$ \\
\hline$C(1 \mathrm{~A})$ & $0.1785(1)$ & $0.3392(1)$ & $0.6403(1)$ & $418(5)$ \\
\hline$C(2 A)$ & $0.2348(2)$ & $0.4563(1)$ & $0.6266(1)$ & $500(6)$ \\
\hline$C(3 A)$ & $0.1535(2)$ & $0.5567(1)$ & $0.6555(1)$ & $620(6)$ \\
\hline$C(4 A)$ & $0.0185(2)$ & $0.5416(1)$ & $0.6997(1)$ & $657(7)$ \\
\hline $\mathrm{C}(5 \mathrm{~A})$ & $-0.0371(2)$ & $0.4264(1)$ & $0.7153(1)$ & $584(6)$ \\
\hline$C(6 A)$ & $0.0428(2)$ & $0.3255(1)$ & $0.6842(1)$ & $494(6)$ \\
\hline $\mathrm{C}(1 \mathrm{~B})$ & $0.3498(1)$ & $0.2655(1)$ & $0.5016(1)$ & 421(5) \\
\hline $\mathrm{C}(2 \mathrm{~B})$ & $0.3289(2)$ & $0.3799(1)$ & $0.4081(1)$ & $570(6)$ \\
\hline$C(3 B)$ & $0.4194(2)$ & $0.4079(2)$ & $0.3057(1)$ & $692(7)$ \\
\hline$C(4 B)$ & $0.5298(2)$ & $0.3226(2)$ & $0.2917(1)$ & $680(7)$ \\
\hline$C(5 B)$ & $0.5477(2)$ & $0.2075(2)$ & $0.3829(1)$ & $631(7)$ \\
\hline $\mathrm{C}(6 \mathrm{~B})$ & $0.4604(2)$ & $0.1784(1)$ & $0.4870(1)$ & $509(6)$ \\
\hline$C(1 C)$ & $0.4853(1)$ & $0.1448(1)$ & $0.7706(1)$ & $440(5)$ \\
\hline $\mathrm{C}(2 \mathrm{C})$ & $0.5660(2)$ & $0.2488(1)$ & $0.7022(1)$ & $532(6)$ \\
\hline$C(3 C)$ & $0.6953(2)$ & $0.2245(2)$ & $0.6506(1)$ & $665(7)$ \\
\hline$C(4 C)$ & $0.7468(2)$ & $0.0960(2)$ & $0.6636(1)$ & $749(7)$ \\
\hline$C(5 C)$ & $0.6660(2)$ & $-0.0074(2)$ & $0.7283(1)$ & $703(7)$ \\
\hline$C(6 C)$ & $0.5367(2)$ & $0.0157(1)$ & $0.7818(1)$ & $558(6)$ \\
\hline$C(1 D)$ & $0.3408(1)$ & $0.2544(1)$ & $0.8960(1)$ & $443(5)$ \\
\hline $\mathrm{C}(2 \mathrm{D})$ & $0.4465(2)$ & $0.2679(1)$ & $0.9447(1)$ & $553(6)$ \\
\hline$C(3 D)$ & $0.4279(2)$ & $0.3475(2)$ & $1.0126(1)$ & $735(7)$ \\
\hline$C(4 D)$ & $0.3044(2)$ & $0.4118(2)$ & $1.0335(1)$ & $819(8)$ \\
\hline$C(5 D)$ & $0.1993(2)$ & $0.3977(2)$ & $0.9865(1)$ & $749(7)$ \\
\hline $\mathrm{C}(6 \mathrm{D})$ & $0.2170(2)$ & $0.3206(2)$ & $0.9173(1)$ & $596(6)$ \\
\hline
\end{tabular}

$2 \mathrm{H}, 3,6-\mathrm{H}), 7.50(\mathrm{~s}, 2 \mathrm{H}, \mathrm{NH}), 7.51\left(\mathrm{dd},{ }^{3} \mathrm{~J}=8.1,{ }^{4} \mathrm{~J}=0.8 \mathrm{~Hz}, 2 \mathrm{H}\right.$, 4,5-H). - MS: high resolution $\left[\mathrm{M}^{+}\right], m / z=310.1483$ (calcd. 310.1470); $m / z(\%)=310(100)\left[\mathrm{M}^{+}\right], 218(18), 217(30), 216(10)$.

$$
\begin{array}{ll}
\mathrm{C}_{22} \mathrm{H}_{18} \mathrm{~N}_{2} \text { (310.4) } & \text { Calcd. C } 85.13 \text { H } 5.85 \text { N } 9.03 \\
& \text { Found C } 85.35 \text { H } 6.14 \text { N } 8.84
\end{array}
$$

1-Amino-8-(phenylamino) naphthalene (4): $R_{\mathrm{f}}=0.66$ (dichloromethane); $3.70 \mathrm{~g}(50 \%)$ of colorless platelets from ethanol, m.p. $131^{\circ} \mathrm{C} .-\operatorname{IR}\left(\mathrm{CHCl}_{3}\right): \tilde{v}=3460 \mathrm{~cm}^{-1}\left(\mathrm{NH}_{2}\right), 3370\left(\mathrm{NH}_{2}, \mathrm{NH}\right)$. UV (dioxane): $\lambda_{\max }(\lg \varepsilon)=292 \mathrm{~nm}$ (3.67), 349 (4.06). $-{ }^{1} \mathrm{H}$ NMR $\left(\mathrm{CDCl}_{3}, 360 \mathrm{MHz}\right): \delta=5.00\left(\mathrm{~s}, 2 \mathrm{H}, \mathrm{NH}_{2}\right), 6.30(\mathrm{~s}, 1 \mathrm{H}, \mathrm{NH}), 6.63$ $\left(\mathrm{dd},{ }^{3} J=6.4,{ }^{4} J=2.2 \mathrm{~Hz}, 1 \mathrm{H}, 7-\mathrm{H}\right), 6.88\left(\mathrm{dd},{ }^{3} J=8.5,{ }^{4} \mathrm{~J}=\right.$ $\left.1.0 \mathrm{~Hz}, 2 \mathrm{H}, 2^{\prime}, 6^{\prime}-\mathrm{H}\right), 6.90\left(\mathrm{dd}, 1 \mathrm{H}, 4^{\prime}-\mathrm{H}\right), 7.16\left(\mathrm{dd},{ }^{3} J=7.4,{ }^{4} \mathrm{~J}=\right.$ $1.2 \mathrm{~Hz}, 1 \mathrm{H}, 2-\mathrm{H}), 7.20-7.30(\mathrm{~m}, 2 \mathrm{H}, 3,5-\mathrm{H}), 7.24\left(\mathrm{~m}, 2 \mathrm{H}, 3^{\prime}, 5^{\prime}-\mathrm{H}\right)$, 7.28 (dd, $1 \mathrm{H}, 6-\mathrm{H}), 7.52$ (dd, $\left.{ }^{3} J=8.1,{ }^{4} J=1.2 \mathrm{~Hz}, 1 \mathrm{H}, 4-\mathrm{H}\right)$. MS: high resolution $\left[\mathrm{M}^{+}\right], m / z=234.1180$ (calcd. 234.1157); $m / z$ $(\%)=234(100)\left[\mathrm{M}^{+}\right], 233(17), 219(15), 217(11), 168(7)$.

$$
\begin{aligned}
& \mathrm{C}_{16} \mathrm{H}_{14} \mathrm{~N}_{2} \text { (234.3) Caled. C 82.02 H } 6.02 \text { N } 11.96 \\
& \text { Found C } 82.30 \text { H } 6.12 \text { N } 11.99
\end{aligned}
$$

1,3-Diphenyl-1H-perimidin-2(3H)-one (9): A mixture of $4.5 \mathrm{~g}$ (24 $\mathrm{mmol}$ ) of $1 \mathrm{H}$-perimidin-2(3H)-one $(\mathbf{8})^{[20]}, 15 \mathrm{~g}$ (74 mmol) of iodobenzene, $4.0 \mathrm{~g}$ of copper powder, $4.0 \mathrm{~g}$ of finely divided potassium carbonate, and a trace of iodine was sealed in an ampoule and heated at $240^{\circ} \mathrm{C}$ for $1 \mathrm{~d}$. After cooling the mixture was extracted several times with dichloromethane, and the combined filtered extracts were concentrated under reduced pressure. Chromatography of the residue (silica gel, dichloromethane) afforded $3.60 \mathrm{~g}(44 \%)$ of $9\left(R_{\mathrm{f}}=0.26\right)$ : from methanol colorless platelets, m.p. $193^{\circ} \mathrm{C} .-{ }^{1} \mathrm{H}$ NMR $\left(\mathrm{CDCl}_{3}, 360 \mathrm{MHz}\right): \delta=6.06\left(\mathrm{~d},{ }^{3} J=6.8 \mathrm{~Hz}, 2 \mathrm{H}, 4,9-\mathrm{H}\right)$, $7.17\left(\mathrm{dd},{ }^{3} J \approx{ }^{3} J^{\prime} \approx 7.9 \mathrm{~Hz}, 2 \mathrm{H}, 5,8-\mathrm{H}\right), 7.27\left(\mathrm{dd},{ }^{3} J=8.3,{ }^{4} J=\right.$ $0.7 \mathrm{~Hz}, 2 \mathrm{H}, 6,7-\mathrm{H}), 7.41\left(\mathrm{~d},{ }^{3} J=8.5 \mathrm{~Hz}, 4 \mathrm{H}, 2^{\prime}, 2^{\prime \prime}, 6^{\prime}, 6^{\prime \prime}-\mathrm{H}\right), 7.45(\mathrm{dd}$, $\left.{ }^{3} J \approx{ }^{3} J^{\prime} \approx 7.4 \mathrm{~Hz}, 2 \mathrm{H}, 4^{\prime}, 4^{\prime \prime}-\mathrm{H}\right), 7.55\left(\mathrm{dd},{ }^{3} J \approx{ }^{3} J^{\prime} \approx 7.6 \mathrm{~Hz}, 4 \mathrm{H}\right.$, $\left.3^{\prime}, 3^{\prime \prime}, 5^{\prime}, 5^{\prime \prime}-\mathrm{H}\right)$. - MS: $m / z(\%)=336(100)\left[\mathrm{M}^{+}\right], 288(7), 258(5)$.

$$
\begin{array}{ll}
\mathrm{C}_{23} \mathrm{H}_{16} \mathrm{~N}_{2} \mathrm{O} \text { (336.4) } & \text { Calcd. C 82.12 H } 4.79 \mathrm{~N} 8.33 \\
& \text { Found C } 81.93 \mathrm{H} 4.70 \mathrm{~N} 8.02
\end{array}
$$

Synthesis of 6 by Reductive Ring Cleavage of 9: To a stirred

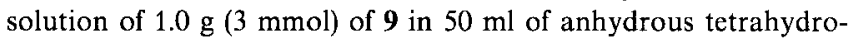
furan (THF) under argon was added dropwise $4 \mathrm{ml}$ of $1.5 \mathrm{~m}(6$ mmol) methyllithium. Stirring was continued for $30 \mathrm{~min}$. After water had been added, the mixture was extracted several times with diethyl ether $(3 \times 100 \mathrm{ml})$. The combined extracts were dried with $\mathrm{MgSO}_{4}$, and the solvent was evaporated under reduced pressure. Chromatography of the residue (silica gel, dichloromethane) afforded $395 \mathrm{mg}(43 \%)$ of $\mathbf{6}$, colorless crystals from ethanol, m.p. $147^{\circ} \mathrm{C}$

1-(Diphenylamino)-8-(phenylamino) naphthalene (7): To $2.0 \mathrm{~g}$ of stirred magnesium turnings in $50 \mathrm{ml}$ of anhydrous THF cooled to $-80^{\circ} \mathrm{C}$ was added under argon $2.0 \mathrm{~g}(11.4 \mathrm{mmol})$ of 1 -bromo-2fluorobenzene. The mixture was slowly heated to reflux. Then a solution of $2.0 \mathrm{~g}(6.4 \mathrm{mmol})$ of 6 in $40 \mathrm{ml}$ of anhydrous THF was added dropwise, and stirring was continued for $30 \mathrm{~min}$. After addition of water the mixture was extracted with diethyl ether $(4 \times$ $100 \mathrm{ml}$ ). The combined extracts were dried with $\mathrm{MgSO}_{4}$, and the solvent was evaporated under reduced pressure. Chromatography of the residue [silicagel, cyclohexane/benzene (3:1)] yielded $720 \mathrm{mg}$ $(29 \%)$ of $7\left(R_{\mathrm{f}}=0.57\right)$, colorless needles from methanol, m.p. $132^{\circ} \mathrm{C}$. - IR $\left(\mathrm{CHCl}_{3}\right): \tilde{v}=3495 \mathrm{~cm}^{-1}(\mathrm{NH}) .-\mathrm{UV}$ (dioxane): $\lambda_{\max }(\lg \varepsilon)$ $=285 \mathrm{~nm}(4.25), 359$ (4.08). $-{ }^{1} \mathrm{H}$ NMR $\left(\mathrm{CDCl}_{3}, 500 \mathrm{MHz}, \mathrm{H}, \mathrm{H}-\right.$ COSY): $\delta=6.91\left(\mathrm{~m}, 1 \mathrm{H}, 4^{\prime \prime \prime}-\mathrm{H}\right), 6.93\left(\mathrm{dd},{ }^{3} J=8.7,{ }^{4} J=1.2 \mathrm{~Hz}\right.$, $\left.2 \mathrm{H}, 2^{\prime \prime \prime}, 6^{\prime \prime \prime}-\mathrm{H}\right), 6.95\left(\mathrm{dd},{ }^{3} J \approx{ }^{3} J^{\prime} \approx 7.4 \mathrm{~Hz}, 2 \mathrm{H}, 4^{\prime}, 4^{\prime \prime}-\mathrm{H}\right), 7.03(\mathrm{dd}$, $\left.{ }^{3} J=8.7,{ }^{4} J=1.0 \mathrm{~Hz}, 4 \mathrm{H}, 2^{\prime}, 2^{\prime \prime}, 6^{\prime}, 6^{\prime \prime}-\mathrm{H}\right), 7.14\left(\mathrm{dd},{ }^{3} J=7.3,{ }^{4} J=\right.$ $1.2 \mathrm{~Hz}, 1 \mathrm{H}, 2-\mathrm{H}), 7.17\left(\mathrm{dd},{ }^{3} \mathrm{~J} \approx{ }^{3} J^{\prime} \approx 7.2 \mathrm{~Hz}, 2 \mathrm{H}, 3^{\prime \prime \prime}, 5^{\prime \prime \prime}-\mathrm{H}\right), 7.19$ (dd, $\left.4 \mathrm{H}, 3^{\prime}, 3^{\prime \prime}, 5^{\prime}, 5^{\prime \prime}-\mathrm{H}\right), 7.20\left(\mathrm{dd},{ }^{3} J=7.8,{ }^{4} J=1.2 \mathrm{~Hz}, 1 \mathrm{H}, 7-\mathrm{H}\right)$, $7.29\left(\mathrm{dd},{ }^{3} J \approx{ }^{3} J^{\prime} \approx 7.8 \mathrm{~Hz}, 1 \mathrm{H}, 6-\mathrm{H}\right), 7.37\left(\mathrm{dd},{ }^{3} J=8.1,{ }^{4} J=\right.$ $1.2 \mathrm{~Hz}, 1 \mathrm{H}, 5-\mathrm{H}), 7.40\left(\mathrm{dd},{ }^{3} J \approx{ }^{3} J^{\prime} \approx 7.7 \mathrm{~Hz}, 1 \mathrm{H}, 3-\mathrm{H}\right), 7.75(\mathrm{dd}$, $\left.{ }^{3} J=8.2,{ }^{4} J=1.2 \mathrm{~Hz}, 1 \mathrm{H}, 4-\mathrm{H}\right), 8.60(\mathrm{~s}, 1 \mathrm{H}, \mathrm{NH})$. - MS: high resolution $\left[\mathrm{M}^{+}\right], m / z=386.1816($ calcd. 386.1783$) ; m / z(\%)=386$ (100) $\left[\mathrm{M}^{+}\right], 294$ (25), 293 (45), 280 (55), 217 (18), 216 (20).

$$
\begin{array}{lll}
\mathrm{C}_{28} \mathrm{H}_{22} \mathrm{~N}_{2} \text { (386.5) } & \text { Calcd. C 87.01 H 5.74 N } 7.25 \\
& \text { Found C 87.10 H 5.94 N } 7.32
\end{array}
$$

1,8-Bis (diphenylamino) naphthalene (2): A mixture of $2.0 \mathrm{~g}(5.2$ $\mathrm{mmol})$ of $7,2.5 \mathrm{~g}(12 \mathrm{mmol})$ of iodobenzene, $0.5 \mathrm{~g}$ of copper powder, $0.5 \mathrm{~g}$ of finely divided potassium carbonate and a trace of iodine was sealed in an ampoule and heated at $240^{\circ} \mathrm{C}$ for $1 \mathrm{~d}$. After cooling the mixture was treated several times with dichloromethane, and the combined filtered extracts were concentrated under reduced pressure. Chromatography of the residue [silica gel, cyclohexane/ benzene (7:3)] yielded two fractions besides iodobenzene:

Fraction 1: $R_{\mathrm{f}}=0.63,220 \mathrm{mg}$, mixture of 2 and 7 as indicated by MS. Separation of the starting material $7(170 \mathrm{mg})$ from this mixture by MPLC by using the reversed-phase material HD-SIL18-30-60 [cyclohexane/benzene (7:3)] yielded $22 \mathrm{mg} \mathrm{(1 \% )} \mathrm{of} \mathrm{2,} \mathrm{col-}$ orless needles from hexane, m.p. $177^{\circ} \mathrm{C}$ - $-\mathrm{UV}$ (dioxane): $\lambda_{\max }(\lg \varepsilon)$ $=293 \mathrm{~nm}$ (4.37), 356 (3.99). $-{ }^{1} \mathrm{H}$ NMR $\left(\mathrm{CDCl}_{3}, 500 \mathrm{MHz}\right): \delta=$ $6.44\left(\mathrm{~d},{ }^{3} J=7.7 \mathrm{~Hz}, 8 \mathrm{H}, 2^{\prime}, 2^{\prime \prime}, 2^{\prime \prime \prime}, 2^{\prime \prime \prime}, 6^{\prime}, 6^{\prime \prime}, 6^{\prime \prime \prime}, 6^{\prime \prime \prime}-\mathrm{H}\right), 6.84\left(\mathrm{dd},{ }^{3} J \approx\right.$ 
$\left.{ }^{3} J^{\prime} \approx 7.3 \mathrm{~Hz}, 4 \mathrm{H}, 4^{\prime}, 4^{\prime \prime}, 4^{\prime \prime \prime}, 4^{\prime \prime \prime}-\mathrm{H}\right), 6.96\left(\mathrm{dd},{ }^{3} J \approx{ }^{3} J^{\prime} \approx 7.5 \mathrm{~Hz}, 8 \mathrm{H}\right.$, $\left.3^{\prime}, 3^{\prime \prime}, 3^{\prime \prime \prime}, 3^{\prime \prime \prime}, 5^{\prime}, 5^{\prime \prime}, 5^{\prime \prime \prime}, 5^{\prime \prime \prime}-\mathrm{H}\right), 6.96\left(\mathrm{dd},{ }^{3} J=7.4,{ }^{4} J=1.0 \mathrm{~Hz}, 2 \mathrm{H}, 2,7-\right.$ H), $7.33\left(\mathrm{dd},{ }^{3} J \approx{ }^{3} J^{\prime} \approx 7.8 \mathrm{~Hz}, 2 \mathrm{H}, 3,6-\mathrm{H}\right), 7.73\left(\mathrm{dd},{ }^{3} J=8.2\right.$, $\left.{ }^{4} J=1.0 \mathrm{~Hz}, 2 \mathrm{H}, 4,5-\mathrm{H}\right)$. $-\mathrm{MS}$ : high resolution $\left[\mathrm{M}^{+}\right], \mathrm{m} / \mathrm{z}=$ $462.2079\left(\mathrm{C}_{34} \mathrm{H}_{26} \mathrm{~N}_{2}\right.$ calcd. 462.2096); $m / z(\%)=462(100)\left[\mathrm{M}^{+}\right]$, 370 (22), 369 (20), 293 (16), 292 (7), 291 (8).

Fraction 2: $R_{\mathrm{f}}=0.51,60 \mathrm{mg}(4 \%)$ of 7-phenyl-7H-benz[kl]acridine (10), yellow crystals from methanol, m.p. $143^{\circ} \mathrm{C} .-{ }^{1} \mathrm{H}$ NMR ( $\left.\left[\mathrm{D}_{6}\right] \mathrm{DMSO}, 500 \mathrm{MHz}\right): \delta=5.64\left(\mathrm{dd},{ }^{3} \mathrm{~J}=6.6,{ }^{4} \mathrm{~J}=2.1 \mathrm{~Hz}\right.$, $1 \mathrm{H}, 6-\mathrm{H}), 6.11\left(\mathrm{~d},{ }^{3} J=8.8 \mathrm{~Hz}, 1 \mathrm{H}, 8-\mathrm{H}\right), 6.95\left(\mathrm{dd},{ }^{3} J \approx{ }^{3} J^{\prime} \approx\right.$ $7.5 \mathrm{~Hz}, 1 \mathrm{H}, 9-\mathrm{H}), 7.02-7.09(\mathrm{~m}, 3 \mathrm{H}, 4,5,10-\mathrm{H}), 7.40\left(\mathrm{dd},{ }^{3} J \approx{ }^{3} J^{\prime}\right.$ $\approx 7.1 \mathrm{~Hz}, 1 \mathrm{H}, 2-\mathrm{H}), 7.41\left(\mathrm{~d},{ }^{3} J=8.3 \mathrm{~Hz}, 2 \mathrm{H}, 2^{\prime}, 6^{\prime}-\mathrm{H}\right), 7.47(\mathrm{~d}$, $\left.{ }^{3} J=8.0 \mathrm{~Hz}, 1 \mathrm{H}, 3-\mathrm{H}\right), 7.63\left(\mathrm{dd},{ }^{3} J \approx{ }^{3} J^{\prime} \approx 7.5 \mathrm{~Hz}, 1 \mathrm{H}, 4^{\prime}-\mathrm{H}\right), 7.75$ $\left(\mathrm{dd},{ }^{3} J=7.9,{ }^{4} J=1.7 \mathrm{~Hz}, 1 \mathrm{H}, 1-\mathrm{H}\right), 7.77\left(\mathrm{dd},{ }^{3} J \approx{ }^{3} J^{\prime} \approx 7.7 \mathrm{~Hz}\right.$, $\left.2 \mathrm{H}, 3^{\prime}, 5^{\prime}-\mathrm{H}\right), 8.02$ (dd, $\left.{ }^{3} J=7.9,{ }^{4} J=1.2 \mathrm{~Hz}, 1 \mathrm{H}, 11-\mathrm{H}\right) .-\mathrm{MS}$ : high resolution $\left[\mathrm{M}^{+}\right], m / z=293.1192$ (calcd. 293.1204); $m / z(\%)=$ $293\left[\mathrm{M}^{+}\right](100), 216(16)$.

$\mathrm{C}_{22} \mathrm{H}_{15} \mathrm{~N}$ (293.4) Calcd. C 90.07 H 5.15 N 4.78

Found C 89.96 H 5.03 N 4.59

1,8-Bis(methylphenylamino) naphthalene (3): To a stirred solution of $1.0 \mathrm{~g}(3.2 \mathrm{mmol})$ of 6 in $50 \mathrm{ml}$ of anhydrous THF was added under argon $4.3 \mathrm{ml}$ of $1.5 \mathrm{M}(6.45 \mathrm{mmol})$ methyllithium. After stirring was continued for $30 \mathrm{~min}, 1.2 \mathrm{~g}(8.5 \mathrm{mmol})$ of methyl iodide was added dropwise. The resulting mixture was stirred for $1 \mathrm{~h}$, then hydrolyzed and extracted with diethyl ether $(2 \times 50 \mathrm{ml})$. The combined organic phases were dried with $\mathrm{MgSO}_{4}$, and the solvent was evaporated under reduced pressure. Chromatography of the residue [silica gel, cyclohexane/benzene (3:1)] yielded $510 \mathrm{mg} \mathrm{(47 \% )}$ of 3 $\left(R_{\mathrm{f}}=0.42\right)$, yellowish platelets from ethanol, m.p. $142^{\circ} \mathrm{C}$. $-\mathrm{UV}$ (dioxane): $\lambda_{\max }(\lg \varepsilon)=311 \mathrm{~nm}(3.81), 346$ (3.77). $-{ }^{\prime} \mathrm{H}$ NMR $\left(\mathrm{CDCl}_{3}, 303 \mathrm{~K}, 500 \mathrm{MHz}\right) 3 \mathrm{a}: \delta=2.71\left(\mathrm{~s}, 6 \mathrm{H}, \mathrm{CH}_{3}\right), 6.40\left(\mathrm{~d},{ }^{3} J=\right.$ $\left.7.9 \mathrm{~Hz}, 4 \mathrm{H}, 2^{\prime}, 2^{\prime \prime}, 6^{\prime}, 6^{\prime \prime}-\mathrm{H}\right), 6.66\left(\mathrm{dd},{ }^{3} J \approx{ }^{3} J^{\prime} \approx 7.2 \mathrm{~Hz}, 2 \mathrm{H}, 4^{\prime}, 4^{\prime \prime}\right.$ H), $7.10\left(\mathrm{dd},{ }^{3} J^{\prime} \approx{ }^{3} J \approx 7.7 \mathrm{~Hz}, 4 \mathrm{H}, 3^{\prime}, 3^{\prime \prime}, 5^{\prime}, 5^{\prime \prime}-\mathrm{H}\right), 7.29\left(\mathrm{dd},{ }^{3} J=\right.$ $\left.7.3,{ }^{4} J=1.1 \mathrm{~Hz}, 2 \mathrm{H}, 2,7-\mathrm{H}\right), 7.48\left(\mathrm{dd},{ }^{3} J \approx{ }^{3} J^{\prime} \approx 7.7 \mathrm{~Hz}, 2 \mathrm{H}, 3,6-\right.$ H), $7.82\left(\mathrm{dd},{ }^{3} J=7.7,{ }^{4} J=1.0 \mathrm{~Hz}, 2 \mathrm{H}, 4,5-\mathrm{H}\right) .-3 \mathrm{a}: \delta=3.30(\mathrm{~s}$, $\left.6 \mathrm{H}, \mathrm{CH}_{3}\right), 6.28\left(\mathrm{~d},{ }^{3} J=8.0 \mathrm{~Hz}, 4 \mathrm{H}, 2^{\prime}, 2^{\prime \prime}, 6^{\prime}, 6^{\prime \prime}-\mathrm{H}\right), 6.4\left(\mathrm{~m}, 2 \mathrm{H}, 4^{\prime}, 4^{\prime \prime}-\right.$ $\mathrm{H}), 6.79\left(\mathrm{dd},{ }^{3} J \approx{ }^{3} J^{\prime} \approx 7.5 \mathrm{~Hz}, 4 \mathrm{H}, 3^{\prime}, 3^{\prime \prime}, 5^{\prime}, 5^{\prime \prime}-\mathrm{H}\right), 7.3(\mathrm{~m}, 2 \mathrm{H}, 2,7-$ H), $7.44\left(\mathrm{dd},{ }^{3} J \approx{ }^{3} J^{\prime} \approx 7.7 \mathrm{~Hz}, 2 \mathrm{H}, 3,6-\mathrm{H}\right), 7.78\left(\mathrm{~d},{ }^{3} J=8.0 \mathrm{~Hz}\right.$, $2 \mathrm{H}, 4,5-\mathrm{H}) .-{ }^{1} \mathrm{H}$ NMR ([D $]$ DMSO, $\left.303 \mathrm{~K}, 500 \mathrm{MHz}\right) 3 \mathrm{a}: \delta=$ $2.61\left(\mathrm{~s}, 6 \mathrm{H}, \mathrm{CH}_{3}\right), 6.28\left(\mathrm{~d},{ }^{3} \mathrm{~J}=8.1 \mathrm{~Hz}, 4 \mathrm{H}, 2^{\prime}, 2^{\prime \prime}, 6^{\prime}, 6^{\prime \prime}-\mathrm{H}\right), 6.62(\mathrm{dd}$, $\left.{ }^{3} J \approx{ }^{3} J^{\prime} \approx 7.2 \mathrm{~Hz}, 2 \mathrm{H}, 4^{\prime}, 4^{\prime \prime}-\mathrm{H}\right), 7.07\left(\mathrm{dd},{ }^{3} J \approx{ }^{3} J^{\prime} \approx 7.9 \mathrm{~Hz}, 4 \mathrm{H}\right.$, $\left.3^{\prime}, 3^{\prime \prime}, 5^{\prime}, 5^{\prime \prime}-\mathrm{H}\right), 7.30\left(\mathrm{dd},{ }^{3} J=6.3,{ }^{4} J=1.1 \mathrm{~Hz}, 2 \mathrm{H}, 2,7-\mathrm{H}\right), 7.58(\mathrm{dd}$, $\left.{ }^{3} J \approx{ }^{3} J^{\prime} \approx 7.8 \mathrm{~Hz}, 2 \mathrm{H}, 3,6-\mathrm{H}\right), 7.95\left(\mathrm{dd},{ }^{3} J=7.4,{ }^{4} J=1.0 \mathrm{~Hz}\right.$, $2 \mathrm{H}, 4,5-\mathrm{H}) .-3 \mathrm{~b}: \delta=3.23\left(\mathrm{~s}, 6 \mathrm{H}, \mathrm{CH}_{3}\right), 6.25\left(\mathrm{~d},{ }^{3} J \approx 8 \mathrm{~Hz}, 4 \mathrm{H}\right.$, $\left.2^{\prime}, 2^{\prime \prime}, 6^{\prime}, 6^{\prime \prime}-\mathrm{H}\right), 6.37\left(\mathrm{dd},{ }^{3} J \approx{ }^{3} J^{\prime} \approx 7.1 \mathrm{~Hz}, \mathrm{~m}, 2 \mathrm{H}, 4^{\prime}, 4^{\prime \prime}-\mathrm{H}\right), 6.75$ (dd, $\left.{ }^{3} J \approx{ }^{3} J^{\prime} \approx 7.9 \mathrm{~Hz}, 4 \mathrm{H}, 3^{\prime}, 3^{\prime \prime}, 5^{\prime}, 5^{\prime \prime}-\mathrm{H}\right), 7.29\left(\mathrm{~d},{ }^{3} J \approx 8 \mathrm{~Hz}, 2 \mathrm{H}, 2,7-\right.$ H), $7.52\left(\mathrm{dd},{ }^{3} J \approx{ }^{3} J^{\prime} \approx 7.8 \mathrm{~Hz}, 2 \mathrm{H}, 3,6-\mathrm{H}\right), 7.88\left(\mathrm{~d},{ }^{3} J=7.8 \mathrm{~Hz}\right.$, $2 \mathrm{H}, 4,5-\mathrm{H}) .-{ }^{1} \mathrm{H}$ NMR ([D $]$ DMSO, $\left.400 \mathrm{~K}, 200 \mathrm{MHz}\right) 3 \mathbf{a}: \delta=$ $6.30\left(\mathrm{~d},{ }^{3} J=7.8 \mathrm{~Hz}, 4 \mathrm{H}, 2^{\prime}, 2^{\prime \prime}, 6^{\prime}, 6^{\prime \prime}-\mathrm{H}\right), 6.57\left(\mathrm{dd},{ }^{3} J \approx{ }^{3} J^{\prime} \approx 7.2 \mathrm{~Hz}\right.$, $\left.2 \mathrm{H}, 4^{\prime}, 4^{\prime \prime}-\mathrm{H}\right), 7.00\left(\mathrm{dd},{ }^{3} J \approx 3^{3} J^{\prime} \approx 7.3 \mathrm{~Hz}, 4 \mathrm{H}, 3^{\prime}, 3^{\prime \prime}, 5^{\prime}, 5^{\prime \prime}-\mathrm{H}\right), 7.29$ (dd, $\left.{ }^{3} J=7.4,{ }^{4} J=1.1 \mathrm{~Hz}, 2 \mathrm{H}, 2,7-\mathrm{H}\right), 7.54\left(\mathrm{dd},{ }^{3} J \approx{ }^{3} J^{\prime} \approx 7.7 \mathrm{~Hz}\right.$, $2 \mathrm{H}, 3,6-\mathrm{H}), 7.90\left(\mathrm{dd},{ }^{3} \mathrm{~J}=8.1,{ }^{4} \mathrm{~J}=1.0 \mathrm{~Hz}, 2 \mathrm{H}, 4,5-\mathrm{H}\right) .-{ }^{13} \mathrm{C}$ NMR $\left(\mathrm{CDCl}_{3}, 303 \mathrm{~K}, 125.8 \mathrm{MHz}\right) \mathbf{3 a}: \delta=41.1\left(2 \mathrm{C}, \mathrm{CH}_{3}\right), 113.1$
$\left(\mathrm{C}-2^{\prime}, 2^{\prime \prime}, 6^{\prime}, 6^{\prime \prime}\right), 116.5\left(\mathrm{C}-4^{\prime}, 4^{\prime \prime}\right), 126.5,127.3,127.5(\mathrm{C}-2,7,-4,5,-3,6)$ $128.6\left(\mathrm{C}-3^{\prime}, 3^{\prime \prime}, 5^{\prime}, 5^{\prime \prime}\right), 137.5$ (C-9), 144.4 (C-1,8), 149.9 (C-1', $\left.1^{\prime \prime}\right)$. $(213 \mathrm{~K}): \delta=41.3\left(2 \mathrm{C}, \mathrm{CH}_{3}\right), 111.1,114.0\left(\mathrm{C}-2^{\prime}, 2^{\prime \prime}, 6^{\prime}, 6^{\prime \prime}\right), 116.1(\mathrm{C}-$ $\left.4^{\prime}, 4^{\prime \prime}\right), 126.4,127.0,127.3(\mathrm{C}-2,7,-4.5,-3,6), 128.3,128.8\left(\mathrm{C}-3^{\prime}, 3^{\prime \prime}, 5^{\prime}, 5^{\prime \prime}\right)$, 136.9 (C-9), 143.6 (C-1,8), $149.1\left(\mathrm{C}-1^{\prime}, \mathbf{1}^{\prime \prime}\right)$. - MS: high resolution $m / z=338.1792$ (calcd. 338.1783; $m / z(\%)=338(100)\left[\mathrm{M}^{+}\right], 246$ (25), 245 (24), 232 (20), 231 (30), 230 (35), 217 (15), 169 (12), 168 (12).

$\mathrm{C}_{24} \mathrm{H}_{22} \mathrm{~N}_{2}$ (338.5) Calcd. C 85.17 H 6.55 N 8.28 Found C 85.15 H 6.73 N 8.15

\section{CAS Registry Numbers}

2: 138052-74-1 / 3: 138052-75-2 / 4: 30407-81-9 / 5: 138052-76-3/ 6: 138052-77-4/ 7: 138052-78-5/8: 5157-11-9/9: 138052-79-6/ 10: $138052-80-9$ / dehydrobenzene: $462-80-6$

${ }^{[1]}$ R. W. Alder, P. S. Bowman, W. R. S. Steele, D. R. Winterman, J. Chem. Soc., Chem. Commun. 1968, 723.

${ }^{[2]}$ H. A. Staab, T. Saupe, Angew. Chem. 1988, 100, 895; Angew. Chem. Int. Ed. Engl. 1988, 27, 865.

${ }^{[3]}$ R. W. Alder, Chem. Rev. 1989, 89, 1215.

${ }^{[4]}$ R. W. Alder, R. B. Sessions in Chemistry of Functional Groups (Ed.: S. Patai), Supplement F, Part 2, Wiley Chichester, 1982, pp. $763-804$

${ }^{[5]}$ R. W. Alder, Acc. Chem. Res. 1983, 16, 321; Tetrahedron 1990, 46,683 .

${ }^{[6]}$ R. W. Alder, J. E. Anderson, J. Chem. Soc., Perkin Trans. 2, $1973,2086$.

[7] T. Herbst, G. P. Schiemenz, C. Wolff, Magn. Reson. Chem. 1988 26, 608 .

${ }^{[8]}$ D. Casarini, L. Lunazzi, G. Placucci, D. Macciantelli, J. Org. Chem. 1987, 52, 4721.

${ }^{[9]}$ D. Casarini, L. Lunazzi, D. Macciantelli, J. Org. Chem. 1988, 53,182 .

${ }^{[10]}$ H. Einspahr, J.-B. Robert, R. E. Marsh, J. D. Roberts, Acta Crystallogr., Sect. B, 1973, 29, 1611.

${ }^{[11]}$ H. Shanan-Atidi, K. H. Bar-Eli, J. Phys. Chem. 1970, 74, 961.

${ }^{[12]}$ F. A. L. Anet, V. J. Basus, J. Magn. Reson. 1978, 32, 339.

${ }^{[13]}$ H. S. Gutowsky, C. H. Holm, J. Chem. Phys. 1956, 25, 1228.

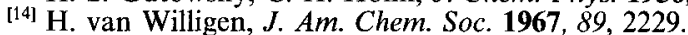

${ }^{[15]}$ F. A. Neugebauer, S. Bamberger, W. R. Groh, Chem. Ber. 1975, 108,2406

${ }^{[16]}$ T. Barth, C. Krieger, F. A. Neugebauer, H. A. Staab, Angew. Chem. 1991, 103, 1006; Angew. Chem. Int. Ed. Engl. 1991, 30 , 1028 , and unpublished results.

${ }^{[17]}$ Further information concerning the X-ray structure analysis of 2 including the tables of atomic coordinates, thermal parameters, bond lengths as well as bond and torsional angles may be requested from Fachinformationszentrum Karlsruhe, Geseilschaft für wissenschaftlich-technische Information mbH, D-7514 Eggenstein-Leopoldshafen 2, with indication of the registry number CSD-55863, the names of the authors, and the reference to this publication.

${ }^{[18]}$ B. A. Frenz and Associates Inc., Structure Determination Package, 4th revised edition, College Station, Texas 77840, USA, and Enraf-Nonius, Delft, The Netherlands, 1982; P. Main, S. J. Fiske, S. E. Hull, L. Lessinger, G. Germain, J.-P. Declercq, M. M. Woolfson, MULTAN80, 1980. Univs. of York, England, and Louvain, Belgium, 1980.

[19] International Tables for $X$-Ray Crystallography, vol. IV, Kynoch Press, Birmingham, 1974.

${ }^{\left[{ }^{20]}\right.}$ F. Sachs, Liebigs Ann. Chem. 1909, 365, 53, p. 136. 